\title{
A intervenção humor em enfermagem: uma revisão de escopo
}

Paulo César Lopes Silva ULSBA, ICS/UCP

Luís Manuel Mota de Sousa CHRC, UE

Cristina Maria Alves Marques-Vieira CIIS, ICS/UCP

\section{Helena Maria Guerreiro José} UICISA:E, UALG 


\section{RESUMO}

Objetivo: Identificar a produção científica, nos últimos 3 anos, sobre o uso do humor nos cuidados de enfermagem prestados a pessoas adultas. Método: Revisão escopo segundo a metodologia de Arksey e O'Malley. Foram pesquisados artigos publicados entre 2018 e 2021, nas plataformas EBSCO Host, Biblioteca Virtual em Saúde e Google Académico. Resultados: Foram identificados 124 artigos e incluídos 11 destes. Foi encontrada informação sobre o humor e a relação entre o paciente e enfermeiro; a viabilidade e usabilidade do humor como intervenção; os benefícios do humor na saúde dos pacientes; os benefícios do humor na saúde dos enfermeiros; os fatores que influenciam o humor; as precauções na utilização do humor; as implicações para a prática e educação em enfermagem. Conclusão: O humor apresenta um carácter pessoal e paradoxal. O enfermeiro deve ter em consideração os fatores associados e os que o influenciam, nas diversas etapas do processo de enfermagem. É uma intervenção terapêutica de baixa intensidade que deve ser usada com precaução.

Palavras-chave: Comunicação, Relações Enfermeiro-Paciente, Senso de Humor e Humor Como Assunto, Enfermagem. 


\section{- INTRODUÇÃO}

O humor tem sido objeto de pesquisas e reflexões filosóficas há séculos, assim como tem sido utilizado como intervenção no setor saúde (LINGE-DAHL et al., 2018).

O humor por meio das experiências e expressões interessantes permite proporcionar diversão, estimulando uma descoberta lúdica na vida cotidiana (ASSOCIATION FOR APPLIED AND THERAPEUTIC HUMOR, 2010).

O modelo teórico do efeito do humor na saúde foi descrito extensivamente por alguns teóricos, e concluíram que o humor tem de ser visto como um fenômeno psicológico complexo que precisa ser diferenciado de acordo com o tipo de humor e o ambiente onde é utilizado (LINGE-DAHL et al., 2018).

O humor e riso estão associados, são influenciados mutuamente, mas não são sinônimos, mas também não podem ser discutidos separadamente. O humor é uma resposta emocional e pode ser manifestada por alegria, enquanto o riso corresponde a uma faceta comportamental. Tanto o riso como o humor são influenciados por um contexto ou situação (SOUSA; JOSÉ, 2016; SOUSA et al., 2018a; SOUSA et al., 2019).

Em contexto de cuidados de enfermagem, o humor é convidado como intervenção e como recurso na Classificação internacional para a Prática de Enfermagem (CIPE ${ }^{\circledR}$ ) e como intervenção Humor (5320) com 15 atividades na Classificação de intervenções em enfermagem [Nursing, Intervention Classification, NIC] (BUTCHER et al., 2018). E surge com intervenção sugerida em alguns diagnósticos de enfermagem como por exemplo ansiedade, dor e tensão do papel de cuidador (JOHNSON et al., 2013).

Estudos realizados na área do humor como intervenção de enfermagem têm demonstrado que o humor é benéfico para a saúde e o bem-estar das pessoas em contexto de doença (LINGE-DAHL et al., 2018; SOUSA; JOSÉ, 2016; SOUSA et al., 2018a; SOUSA et al., 2019a).

O uso do humor favorece a comunicação, que é essencial para as interações humanas. Ajuda a promover o bem-estar; lidar com situações difíceis e desagradáveis, reduzindo a tensão, o estresse e o desconforto; aumenta a tolerância à dor e fortalece o sistema imunológico (SOUSA et al., 2018a; SOUSA et al., 2019a).

O humor é uma intervenção de enfermagem complexa, que deve ser usada quando apropriado. Contudo, requer cuidados, treinamento e o enfermeiro tem de adaptar as atividades da intervenção humor a cada pessoa (SOUSA et al., 2019). Neste sentido, seria importante verificar se existem atualizações nas pesquisas no âmbito da intervenção humor em enfermagem. 


\section{- OBJETIVOS}

Identificar a produção científica, nos últimos 3 anos, sobre os fatores que influenciam o uso do humor nos cuidados de enfermagem e sua aplicabilidade; e ainda, os benefícios da intervenção do humor em enfermagem na saúde e bem-estar em pessoas adultas.

\section{- MÉTODOS}

\section{Aspetos éticos}

Trata-se de um estudo secundário, por isso, não envolve diretamente as pessoas, contudo, os procedimentos éticos foram garantidos ao nível do rigor metodológico, na referenciação e respeito pelas ideias dos autores.

\section{Projeto, local do estudo e período}

Foi utilizada a metodologia de revisão de escopo. Este tipo de revisão é definido como uma técnica para "mapear" literatura relevante na área de interesse. Consiste em cinco etapas: identificação da questão de pesquisa; identificar estudos relevantes; seleção de estudos; mapear os dados; e compilar, resumir e relatar os resultados (ARKSEY; O'MALLEY, 2005; SOUSA et al., 2018b).

A pesquisa eletrônica foi realizada em março de 2021 utilizando a plataforma EBSCO Host $^{\boxplus}$ (CINAHL Complete e MEDLINE Complete), a Biblioteca Virtual de Saúde (BVS), o Google Scholar e o Repositório Científico de Acesso Aberto de Portugal (RCAAP).

\section{Protocolo de estudo}

A questão inicial foi definida a partir do framework - Paciente $(P)$, Conceito $(C$ [1]) e Contexto (C [2]) (ARKSEY; O'MALLEY, 2005). Nesse sentido, a questão de pesquisa foi a seguinte: que conhecimento produzido nos últimos 3 anos sobre integração da intervenção humor (C [1]) em contexto de enfermagem (C [2]) nas pessoas adultas em situação de doença $(P)$ ?

Os descritores foram validados na plataforma de Descritores em Ciências da Saúde (DeCS). Foram utilizadas outras palavras-chave, além dos descritores.

As equações booleanas foram utilizadas:

S1: AB ((Humor therapy) OR (Humor) OR (Wit and Humor as Topic))

S2: AB ((Nursing Care) OR (nursing Intervention) OR (Nurs $\left.\left.{ }^{\star}\right)\right)$

S3: AB ((S1) AND (S2)) 
A pesquisa foi conduzida de forma independente por dois pesquisadores da equipe de pesquisa, e a seleção dos estudos seguiu o mesmo método, com a sequência sugerida por diretrizes internacionais (TRICCO et al., 2018). Os resultados foram obtidos de forma faseada, desde a leitura do título até a leitura do texto completo.

\section{Amostras, critérios de inclusão e exclusão}

Foram incluídos artigos completos publicados nos últimos 3 anos, nos idiomas português, inglês, francês e espanhol, que abordassem o uso da intervenção humor em contexto de cuidados de enfermagem, fatores de influenciam o humor em saúde, cuidados a ter com a intervenção e benefícios dessa na saúde e bem-estar de pessoas adultas em processo de doença.

\section{Extração e Análise dos Resultados}

Após a seleção dos artigos, estes foram analisados e em seguida foram coletadas informações sobre autor(es), ano de publicação, local do estudo, população (grupo que recebeu a intervenção humor em enfermagem), objetivos do estudo, metodologia e resultados importantes.

O nível de evidência foi classificado tendo por base as diretrizes da Registered Nurses' Association of Ontario (RNAO): la - Evidências obtidas em metanálise ou revisão sistemática de estudos randomizados controlados lb - Evidências obtidas em pelo menos um ensaio clínico randomizado controlado lla - Evidências obtidas de pelo menos um estudo controlado bem delineado sem randomização Ilb - Evidências obtidas em pelo menos um outro tipo de estudo quase experimental bem delineado III - Evidências obtidas de um estudo descritivo não experimental bem delineado, como estudo comparativo, caso ; IV - evidências obtidas em relatórios de comitês de especialistas ou opiniões e / ou experiências clínicas de autoridades respeitadas (SOUSA et al., 2017).

Todo o processo que teve início com identificação, seleção, elegibilidade, inclusão dos estudos e de extração de dados, culminou na construção do quadro-resumo de cada artigo com a sistematização das informações relevantes foi feito de modo independente por dois pesquisadores.

\section{- RESULTADOS}

Um total de 124 artigos foram identificados. Após remoção de registos duplicados, foram obtidos 62 artigos. Esses artigos foram submetidos a leitura do título e resumo, sendo selecionados 17 artigos para análise do texto completo. Seis artigos foram excluídos após 
essa análise, sendo apontados os motivos de exclusão: três artigos não respondiam à questão de investigação, dois artigos não cumpriram os critérios de inclusão, tendo em conta o idioma, um artigo não apresentava texto integral. A amostra foi constituída por 11 artigos que cumpriram os critérios de inclusão e foram selecionados para revisão final (Figura 1).

Figura 1. Prisma Flowchart da seleção de estudos para a revisão de escopo.
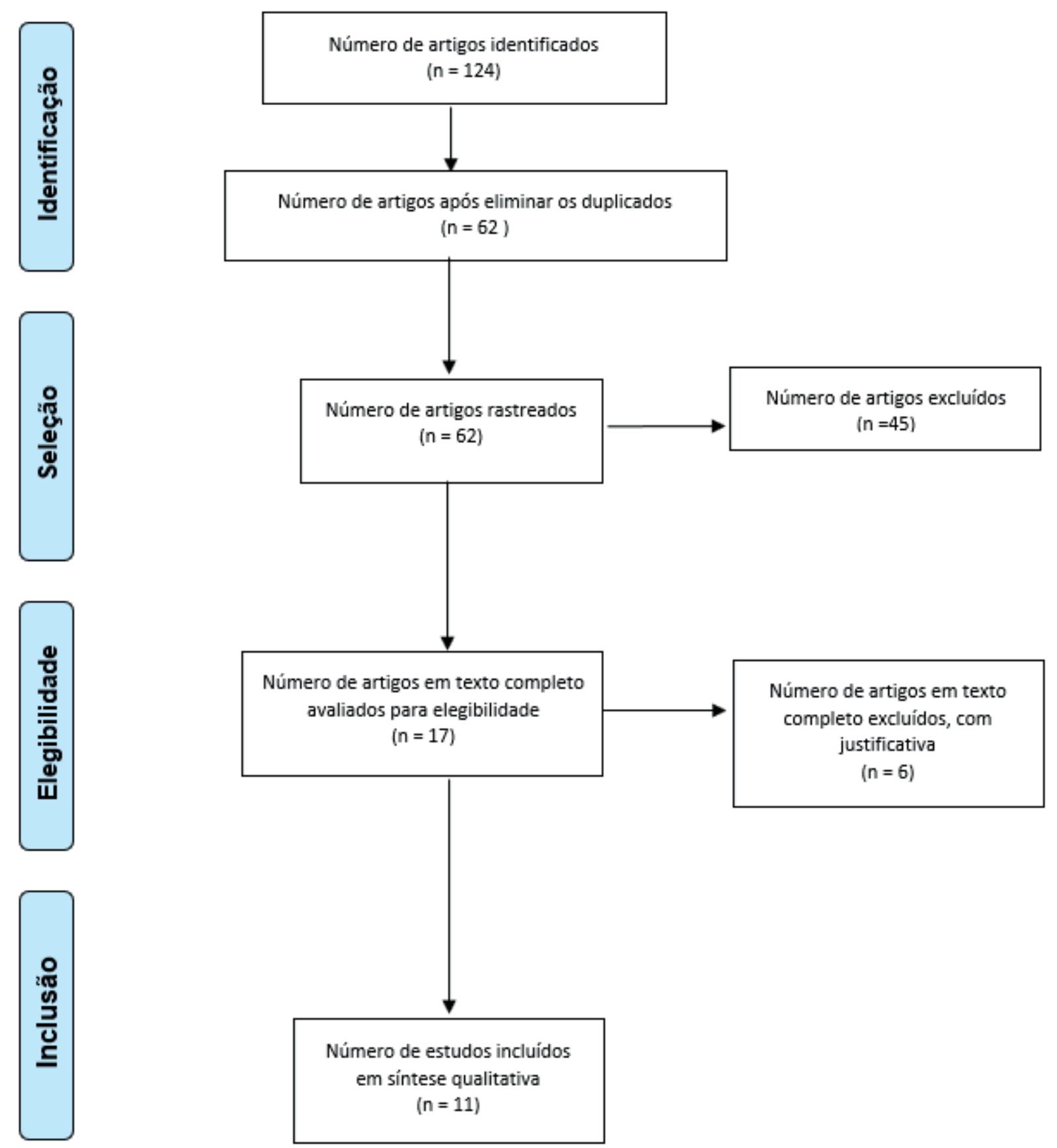

Foram incluídos estudos de abordagem qualitativa e quantitativa. No que concerne aos estudos qualitativos, foram incluídos: uma revisão de escopo (SOUSA et al, 2019a), dois estudos descritivos (VAN DER KROGT et al., 2020; TORRES-VIGIL et al., 2021), uma revisão sistemática da literatura (MACEDO et al., 2019) e uma revisão narrativa da literatura (FIGUEIREDO et al., 2020). No âmbito dos estudos quantitativos foram analisados: uma 
metanálise de ensaios randomizados controlados (ZHAO et al., 2019); dois estudos quasi-experimentais (SOUSA et al, 2019b; ZHAO et al., 2020), um ensaio clínico randomizado controlado (BERHOU et al, 2019), um estudo correlacional (NUNES et al., 2018) e um estudo pré-teste/pós-teste (SOUSA et al., 2019c). Assim, quanto à classificação da amostra da revisão de escopo, tendo em conta o nível de evidência, e de acordo as diretrizes da RNAO, pode aferir-se que: um estudo apresenta nível de evidência la (ZHAO et al., 2019); um estudo apresenta nível de evidência lb (BERHOU et al., 2019); três estudos apresentam nível de evidência lla (SOUSA et al, 2019b; SOUSA et al., 2019c; ZHAO et al., 2020); quatro estudos apresentam nível de evidência III (MACEDO, et al., 2019; NUNES et al. 2018; VAN DER KROGT et al., 2020; TORRES-VIGIL et al., 2021) e dois apresenta nível de evidência IV (FIGUEIREDO et al., 2020; SOUSA et al, 2019a).

Os artigos foram publicados no seguinte espectro temporal: um artigo em 2018 (NUNES et al., 2018), seis artigos em 2019 (BERHOU et al, 2019; SOUSA et al, 2019a; SOUSA et al, 2019b; SOUSA et al., 2019c; ZHAO et al., 2019; MACEDO, et al., 2019) e três artigos em 2020 (FIGUEIREDO et al., 2020; VAN DER KROGT et al., 2020; ZHAO et al., 2020). Apenas foi publicado um artigo, no ano corrente (TORRES-VIGIL et al., 2021), que cumpre os critérios de elegibilidade.

Com cinco (MACEDO, et al., 2019; NUNES et al., 2018; FIGUEIREDO et al., 2020; SOUSA et al., 2019a; SOUSA et al., 2019b; SOUSA et al., 2019c) artigos publicados, Portugal destaca-se como o país de origem do maior número de publicações nos anos a que se reporta a revisão. Segue-se a China, com dois artigos (ZHAO et al., 2019; ZHAO et al., 2020) e com menor expressão surgem outros países: Nova Zelândia (VAN DER KROGT et al., 2020), Irã (BERHOU et al, 2019), EUA (TORRES-VIGIL et al., 2021) e Brasil (FIGUEIREDO et al., 2020).

Os artigos analisam a aplicação do humor enquanto estratégia terapêutica e destacam os benefícios para os intervenientes. São estudados contextos específicos, tais como: unidades de hemodiálise (MACEDO, et al., 2019; SOUSA et al, 2019a; SOUSA et al, 2019b; SOUSA et al., 2019c), lares de idosos (ZHAO et al., 2020), unidade cirúrgica (VAN DER KROGT et al., 2020) e cuidados paliativos (NUNES et al. 2018; TORRES-VIGIL et al., 2021).

As características dos artigos analisados encontram-se presentes no quadro 1.

Os temas que emergiram da análise dos resultados dos artigos incluídos forram: 1) o humor e a relação entre o paciente e enfermeiro (BERHOU et al, 2019; NUNES et al. 2018; FIGUEIREDO et al., 2020; SOUSA et al, 2019a; TORRES-VIGIL et al., 2021; VAN DER KROGT et al., 2020; ZHAO et al., 2019); 2) viabilidade e usabilidade do humor como intervenção (MACEDO, et al., 2019; FIGUEIREDO et al., 2020; SOUSA et al., 2019c; TORRESVIGIL et al., 2021; VAN DER KROGT et al., 2020; ZHAO et al., 2019; ZHAO et al., 2020); 3) benefícios do humor na saúde dos pacientes (BERHOU et al, 2019; MACEDO et al., 
2019; SOUSA et al, 2019a; SOUSA et al., 2019b; SOUSA et al., 2019c; ZHAO et al., 2019;

ZHAO et al., 2020); 4) benefícios do humor na saúde dos enfermeiros (FIGUEIREDO et al., 2020); 5) fatores que influenciam o humor (SOUSA et al, 2019a; SOUSA et al, 2019b); 6) precauções na utilização do humor (SOUSA et al, 2019a) e 7) implicações para a prática e educação em enfermagem (VAN DER KROGT et al., 2020; ZHAO et al., 2020).

Quadro 1. Caracteristicas dos artigos analisados.

\begin{tabular}{|c|c|c|c|c|}
\hline $\begin{array}{l}\text { Autor(s), Ano, } \\
\text { Local }\end{array}$ & População & Objetivo & Método & Resultados \\
\hline
\end{tabular}

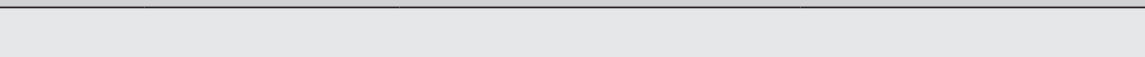

A heterogeneidade foi elevada em todos os estudos combinados $\left(p=0,09, \mathrm{I}^{2}=64 \%\right)$.

Foram detetados efeitos positivos do riso e interven-

Quantitativo;

10 ensaios rando- Descrever o efeito das intervenZHAO et al., 2019, mizados controla- ções de riso e humor na depresChina dos que incluem são, ansiedade e qualidade do 814 participantes sono em adultos.
Metanálise de ensaios randomizados controlados; Recurso ao Cochrane Collaboration bias assessment tool.
SOUSA et al, 2019a, Portugal
17 estudos

Descrever os fatores que influenciam o uso do humor na assistência de enfermagem, sua aplicabilidade e benefícios.
Qualitativo; Revisão de escopo.
67 pessoas com doença renal crônica

SOUSA et al, 2019b, Portugal programa de hemodiálise

I $(n=34)$

$C(n=33)$
Avaliar a efetividade da visualização de filmes de humor na saúde psicológica, bem-estar subjetivo, interferência da dor e qualidade de vida em pessoas com doença renal crônica durante as sessões de hemodiálise.
Quantitativo;

Estudo quasi-experimental. ções de humor na depressão, ansiedade e qualidade do sono em adultos comparativamente com o tratamento desprovido de tais intervenções.

2 estudos analisados demonstraram que a aplicação de humor e riso pode proporcionar um ambiente interpessoal harmonioso e fazer com que as pessoas se sintam seguras.

O recurso ao humor e ao riso pode promover relações interpessoais harmoniosas entre os pacientes e, concomitantemente, pode melhorar o relacionamento enfermeiro-paciente e promover a adesão ao tratamento.

O uso do humor favorece a comunicação, a qual desempenha um papel preponderante nas interações humanas. Auxilia na promoção do bem-estar; permite lidar com situações difíceis e desagradáveis, reduzindo a tensão, o stress e o desconforto; aumenta a tolerância à dor, e fortalece o sistema imunológico.

É influenciado por fatores internos (personalidade) e externos (sociais). O enfermeiro deve utilizá-lo com cautela, implica ainda, treino e a adoção de estratégias individualizadas.

O grupo de intervenção apresentou maiores valores percentuais de mudança no estresse e ansiedade $(-20,6 \%)$, na depressão $(-43,2 \%)$ e na atitude em relação ao humor $(9,8 \%)$.

Este estudo veio demonstrar evidência sobre a visualização de filmes de humor na melhoria do sentido de humor, do bem-estar subjetivo, da qualidade de vida e na diminuição dos sintomas depressivos na pessoa com doença renal crônica submetida a programa de hemodiálise. A prescrição de filmes de humor pelo enfermeiro demonstra ser uma intervenção de baixa intensidade que traz benefícios na saúde e na qualidade de vida dessas pessoas.

O grupo de intervenção demonstrou sintomas de depressão significativamente mais baixos $(p<0,05)$, uma redução significativa da ansiedade $(p<0,05)$ e um score mais elevado na The Memorial University of Newfoundland Scale of Happiness (MUNSH) $(p<0,01)$ comparando com o grupo de controlo. Na aplicação do Pittsburgh Sleep Quality Index (PSQI) o escore de qualidade do sono foi significativamente reduzido desde o início da intervenção até ao período de follow-up (16 semanas) $(p<0,01)$.

Quantitativo; 8 sema intervenção humor, com são, ansiedade, bem-estar subjetivo, função cognitiva e qualidade do sono entre residentes de lares de idosos.
Estudo quasi-experimental.
Estes achados demonstram que a intervenção humor poderá ser viável e bem recebida, assim como pode reduzir os sintomas de depressão e ansiedade e melhorar o bem-estar subjetivo, função cognitiva e qualidade do sono em idosos institucionalizados. Estes benefícios parecem manter-se no período pós-intervenção. Assim, os enfermeiros podem recorrer à intervenção humor como uma abordagem não farmacológica na melhoria contínua dos cuidados. 


\begin{tabular}{|c|c|c|c|c|}
\hline $\begin{array}{l}\text { Autor(s), Ano, } \\
\text { Local }\end{array}$ & População & Objetivo & Método & Resultados \\
\hline
\end{tabular}

Foi demonstrado que os enfermeiros recorrem ao humor de forma consciente e com intencionalidade terapêutica. O humor tem sido utilizado por enfermeiros para proporcionar conforto à pessoa, a si próprios e à

VAN DER KROGT et al., 2020, Nova Zelândia

9 enfermeiros

Explorar o uso do humor por enfermeiros num ambiente cirúrgico.
Qualitativo;

Estudo descritivo;

Entrevistas semi-estruturadas. equipa de enfermagem. O humor também foi usado intencionalmente para iniciar e manter a relação enfermeiro-pessoa. Promover o humor como estratégia de comunicação de enfermagem significa um desafio às crenças de que o humor não é profissional ou impróprio.

Os achados apontam para o humor como uma competência clínica e sugerem a sua incorporação em guidelines e nos currículos de ensino.

Após a intervenção ocorreu diminuição significativa da dor $(p<0,001)$ e do medo da dor $(p<0,05)$. Esses resultados não foram significativos no grupo controle

Determinar o impacto da terapia do humor na qualidade e no medo da dor em idosos institucionalizados através da implementação de um programa de intervenção com a duração de 6 sessões.

\section{Quantitativo:}

Ensaio clínico randomizado controlado.

\section{( $p>0,05)$.}

A terapia do humor demonstra eficácia como intervenção complementar aos cuidados de enfermagem, com impacto na dimensão cognitiva da dor e demonstra ter um efeito positivo na redução da qualidade e do medo da dor em idosos institucionalizados.
NUNES et al. 2018, Portugal 66 enfermeiros de humor e o luto profissional em enfermeiros que trabalham em cuidados paliativos.
Quantitativo;

Estudo correlacional.

Os enfermeiros obtiveram pontuações mais altas do que as enfermeiras no âmbito da produção e uso social do humor ( $p<0,05)$, assim como ao nível do humor de enfrentamento $(p<0,01)$. Enfermeiros com educação específica em cuidados paliativos apresentaram maior senso de humor $(p<0,05)$ e maior capacidade para o uso social do humor $(p<0,05)$.

Uma atitude positiva em relação ao humor parece facilitar o trabalho emocional dos enfermeiros na prestação de cuidados a pessoas em fim-de-vida.

Foram identificados três temas principais em relação à empatia: Compreender as experiências relatadas; Comunicar a compreensão das experiências relatadas (subtemas: humor e validação); Agir em conformidade com as experiências relatadas (subtemas: resolução de problemas e fornecimento de suporte).

Qualitativo;

Descrever a natureza e os eleTORRES-VIGIL et al., 2021, EUA
95 pessoas com câncer em estadio avançado follow-up chave de chamadas de meiros para pessoas com câncer em estadio avançado.
Estudo descritivo;

Análise temática segundo Braun \& Clark As chamadas telefônicas empáticas realizadas por enfermeiros são uma abordagem simples, viável e acessível que pode melhorar os resultados em pessoas com câncer avançado.

O grupo de intervenção apresentou um aumento de $1.6 \%$ na componente física da QV, 3.6\% na componente mental da QV e diminuição de $3.2 \%$ na severidade da dor, assim como de $6.1 \%$ na interferência da dor. No grupo de controlo verificou-se uma diminuição de cerca de $10 \%$ na componente física da QV, de $19.1 \%$ na interferência na dor e de $5.9 \%$ na severidade da dor. Aumentou cerca de 5.8\% na componente mental da QV. É evidente o caráter protetor da visualização de filmes de humor sobre a componente física da QV, constatando-se diminuições significativas dos valores da pressão arterial sistólica $(p<0,05)$ e pressão arterial diastólica $(p<0,05)$ no grupo de intervenção. 2019c, Portugal
SOUSA et al.,
70 pessoas com dohemodiálise

I $(n=34)$

$C(n=33)$ ença renal crônica
Avaliar os benefícios da visualização de filmes de humor na qualidade de vida (QV) das pessoas com DRC em programa de hemodiálise pré-teste/pó-teste grupo controle não equivalente. 


\begin{tabular}{|c|c|c|c|c|}
\hline $\begin{array}{l}\text { Autor(s), Ano, } \\
\text { Local }\end{array}$ & População & Objetivo & Método & Resultados \\
\hline $\begin{array}{l}\text { MACEDO,et al., } \\
2019 \\
\text { Portugal }\end{array}$ & $\begin{array}{c}5 \text { estudos quasi-ex- } \\
\text { perimentais }\end{array}$ & $\begin{array}{l}\text { Destacar os benefícios na saúde } \\
\text { e bem-estar das intervenções de } \\
\text { Enfermagem indutoras do riso e } \\
\text { do humor em pessoas submetidas } \\
\text { a hemodiálise }\end{array}$ & $\begin{array}{c}\text { Qualitativo; } \\
\text { Revisão Sistemática da } \\
\text { Literatura. }\end{array}$ & $\begin{array}{l}\text { A exibição de filmes, o yoga do riso e a terapia do riso } \\
\text { simulado aparecem como as principais intervenções } \\
\text { indutoras do riso e humor. } \\
\text { Assistir a filmes de humor e praticar ioga do riso diminui } \\
\text { a pressão arterial, sugerem ser prescrições de baixa } \\
\text { intensidade e custo. } \\
4 \text { dos } 5 \text { estudos relatam efeitos benéficos sobre a de- } \\
\text { pressão, ansiedade e estresse, existindo uma clara di- } \\
\text { minuição dos níveis desses componentes. } \\
\text { São recomendados mais estudos no âmbito da QV e } \\
\text { bem-estar subjetivo devido à falta de consenso entre } \\
\text { os estudos analisados. }\end{array}$ \\
\hline $\begin{array}{l}\text { FIGUEIREDO et } \\
\text { al., 2020, Brasil }\end{array}$ & $\begin{array}{l}\text { Teses, dissertações } \\
\text { e artigos científi- } \\
\text { cos publicados no } \\
\text { período de } 2007 \text { a } \\
2017\end{array}$ & $\begin{array}{l}\text { Compreender a natureza do hu- } \\
\text { mor na prática de enfermagem e } \\
\text { descrever as suas manifestações } \\
\text { e benefícios. }\end{array}$ & $\begin{array}{c}\text { Qualitativo; } \\
\text { Revisão Narrativa. }\end{array}$ & $\begin{array}{l}\text { A literatura analisada descreve que o recurso ao hu- } \\
\text { mor na prática cotidiana de enfermagem pode ser um } \\
\text { método valioso para a pessoa doente. É relatado um } \\
\text { efeito positivo na saúde dos mesmos, por melhorar a } \\
\text { comunicação entre os intervenientes e permitir a re- } \\
\text { dução dos níveis de estresse e exaustão que acomete } \\
\text { grande parte dos enfermeiros }\end{array}$ \\
\hline
\end{tabular}

\section{- DISCUSSÃO}

A discussão foi estruturada de acordo com diversas categorias definidas após a leitura dos artigos selecionados, são elas: o humor e a relação entre o paciente e enfermeiro; viabilidade e usabilidade do humor como intervenção; benefícios do humor na saúde dos pacientes; benefícios do humor na saúde dos enfermeiros; fatores que influenciam o humor; precauções na utilização do humor; implicações para a prática e educação em enfermagem.

\section{O humor e a relação entre o paciente e enfermeiro}

O uso do humor favorece a comunicação, a qual desempenha um papel preponderante nas interações humanas (SOUSA et al., 2019a; TORRES-VIGIL et al., 2021). O humor, assim como o riso, é uma estratégia terapêutica que parece promover a harmonia na relação entre pacientes (ZHAO et al., 2019), o relacionamento enfermeiro-paciente (ZHAO et al., 2019; SOUSA et al., 2019a; VAN DER KROGT et al., 2020 TORRES-VIGIL et al, 2021; FIGUEIREDO et al, 2020) assim como sugere funcionar como agente coadjuvante da adesão ao tratamento implementado (ZHAO et al., 2019). Tem sido usado intencionalmente para despoletar e manter a relação enfermeiro-pessoa (SOUSA et al., 2019a; SOUSA et al., 2019b; SOUSA et al., 2019c; VAN DER KROGT et al., 2020).

Uma atitude positiva em relação ao humor tem sido amplamente abordada (NUNES et al, 2018; FIGUEIREDO et al., 2020; TORRES-VIGIL et al., 2021). Tal atitude parece facilitar o trabalho emocional dos enfermeiros, nomeadamente na prestação de cuidados a pessoas em fim-de-vida (NUNES et al. 2018). Neste contexto, o recurso ao humor demonstrando compreender as experiências relatadas pela pessoa, envolvência e agindo em conformidade com as experiências relatadas são estratégias simples, viáveis e acessíveis que aproximam 
o enfermeiro do paciente (TORRES-VIGIL et al., 2021). Assim, o humor é considerado como um fator protetor da relação iniciada pelos pacientes, ou seja, por meio do humor, eles expressam mensagens sérias e lidam com questões emocionais mais facilmente (SCHÖPF; MARTIN; KEATING, 2017).

A promoção do humor enquanto estratégia de comunicação de enfermagem representa um desafio a crenças enraizadas de que o humor é impróprio para ser empregado no contexto dos cuidados de saúde e uma intervenção de cariz não profissional (VAN DER KROGT et al., 2020).

O humor pode ser assim utilizado para melhorar a experiência do paciente durante o internamento, nivelando a relação enfermeiro-paciente (SOUSA; JOSÉ, 2016; SOUSA et al., 2018a; SOUSA et al., 2019a).

\section{Viabilidade e usabilidade do humor como intervenção}

Os estudos analisados são consensuais quanto à empregabilidade e utilidade do humor na enfermagem, nomeadamente na população idosa e em situação de doença. Estudos experimentais desenvolvidos com indivíduos adultos demonstram que o humor proporciona um ambiente interpessoal harmonioso que pode incrementar a segurança da pessoa cuidada (ZHAO et al., 2019).

Em idosos institucionalizados, a intervenção humor poderá ser viável e bem recebida (ZHAO et al., 2019; ZHAO et al., 2020). O humor faz parte da interação paciente-enfermeiro e permite melhorar a comunicação com os idosos (KRISTENSEN et al., 2017). O recurso ao humor como intervenção de enfermagem sugere ser uma abordagem não farmacológica na melhoria contínua dos cuidados (BERHOU et al, 2019; ZHAO et al., 2020).

Um estudo randomizado controlado demonstra a eficácia do humor como intervenção complementar aos cuidados de enfermagem (BERHOU et al, 2019). A exibição de filmes (SOUSA et al., 2019b; SOUSA et al., 2019c), o yoga do riso e a terapia do riso simulado surgem como as principais intervenções indutoras do riso e humor (MACEDO et al., 2019). Apresentam-se como prescrições de enfermagem de baixa intensidade e custo (SOUSA et al, 2019b; MACEDO et al, 2019). As intervenções de baixa intensidade e custo, referem-se ao tempo e aos recursos que são utilizados durante a implementação e neste sentido, a intervenção humor é considerada de baixa intensidade (KREITZER; KOITHAN, 2014).

Considerada uma abordagem subjetiva e não intencional, é frequente o humor surgir de forma espontânea na relação enfermeiro-paciente. Contudo, estudos recentes referem que os enfermeiros começam a recorrer ao humor de forma consciente e com intencionalidade terapêutica (Sousa et al., 2019a; Sousa et al., 2019b; Sousa et al., 2019c; van der Krogt et al., 2020). 
O humor apresenta-se como uma intervenção viável na abordagem a populações frágeis. Um estudo desenvolvido junto de pacientes submetidos a programa de hemodiálise revelou o caráter protetor da visualização de filmes de humor (SOUSA et al., 2019C).

Associado ao contexto pandêmico que vivenciamos atualmente, têm sido relatadas novas estratégias como é o exemplo das chamadas telefônicas empáticas (TORRES-VIGIL et al, 2021) que demonstram serem adequadas para promover a adesão terapêutica do paciente e combater a ansiedade.

Uma revisão recente salienta que o humor, na prática cotidiana de enfermagem, poderá ser um método valioso na abordagem à pessoa em situação de doença (FIGUEIREDO et al., 2020). Contudo, existem três critérios para o uso apropriado que devem ser tidos em consideração e que requerem uma avaliação adequada da situação, nomeadamente o momento (timing), a recetividade do paciente (se aprecia o uso de humor) e o conteúdo das deixas de humor (evitar sexismo, racismo, religião e sarcasmo) (HAWKING, 2019).

Além disso, o enfermeiro tem de aprimorar as suas habilidades no uso do humor, tendo em consideração, o contexto, os fatores sociais e culturais em que paciente se encontra (DE ALMEIDA; NUNES, 2020), de modo que a intervenção seja centrada no paciente.

\section{Benefícios do humor na saúde dos pacientes}

A atitude positiva em relação ao humor (NUNES et al, 2018; FIGUEIREDO et al., 2020; TORRES-VIGIL et al., 2021) faz emergir efeitos benéficos do humor na saúde dos pacientes. Estudos experimentais destacam efeitos positivos do riso e intervenções de humor na depressão, ansiedade e qualidade do sono nos adultos em geral (ZHAO et al., 2019).

Evidências anteriores a este estudo demonstram que a prescrição da intervenção humor tem sido eficaz na promoção do bem-estar, no desenvolvimento de estratégias de coping, na redução da tensão, do estresse e do desconforto (ZHAO et al., 2019; SOUSA et al., 2019a; MACEDO et al, 2019) nos pacientes nos mais diversos contextos.

Diversos estudos da abordagem quantitativa demonstram que a capacidade para apreciar o humor aumenta a tolerância à dor (BERHOU et al, 2019; SOUSA et al., 2019b; SOUSA et al., 2019c) e diminui o medo da dor (BERHOU et al, 2019) com elevados níveis elevados de significância.

Evidências apontam para o caráter protetor do humor como intervenção. Tem sido demonstrado que, a visualização de filmes humorísticos (MACEDO et al., 2019; SOUSA et al., 2019c) e a prática de yoga do riso diminui a pressão arterial (MACEDO et al., 2019).

A implementação de um programa de intervenção estruturado, com recurso à visualização de filmes humorísticos, parece fortalecer o sistema imunológico dos pacientes, tal como demonstrado num estudo quasi-experimental desenvolvido junto de pacientes com 
doença renal crônica (SOUSA et al., 2019b). Esse estudo demonstrou que a prescrição de filmes de humor pelo enfermeiro aparenta ser uma intervenção que traz benefícios na saúde e na qualidade de vida dessas pessoas, indicando que podem ser realizados estudos com as mesmas características noutro tipo de populações (SOUSA et al, 2019b).

Estudos quasi-experimentais têm demonstrado que a intervenção humor poderá reduzir os sintomas de depressão e ansiedade (MACEDO et al., 2019; SOUSA et al, 2019b, SOUSA et al, 2019c, ZHAO et al., 2020), melhorar o bem-estar subjetivo (SOUSA et al, 2019b, ZHAO et al., 2020), melhorar a felicidade subjetiva (SOUSA et al, 2019c; SOUSA et al, 2019c; ZHAO et al., 2020), desenvolver a função cognitiva, assim como melhorar a qualidade do sono (ZHAO et al., 2020). Tais efeitos parecem manter-se no período pós-intervenção (ZHAO et al., 2020).

Tem sido estudado o impacto de programas de intervenção do humor na QV. Os dados alcançados apontam para um aumento nas duas componentes da QV (física e mental) (SOUSA et al., 2019c). Contudo ainda não existe consenso entre os diversos estudos desenvolvidos (MACEDO et al, 2019).

Estes resultados vêm reforçar a evidência produzida anteriormente, a utilização do humor permite obter benefícios na saúde e bem-estar dos pacientes, pois ajuda a promover o bem-estar; a lidar com situações difíceis e desagradáveis, a reduzir a tensão, o estresse e o desconforto; aumentar a tolerância à dor, e fortalecer o sistema imunitário (SOUSA; JOSÉ, 2016; SOUSA et al., 2018a).

\section{Benefícios do humor na saúde dos enfermeiros}

Nos últimos anos tem sido discutida a influência do humor na saúde dos profissionais de enfermagem. Um dos estudos analisados (FIGUEIREDO et al., 2020) indica que o recurso a intervenções baseadas no humor conduz à redução dos níveis de estresse e exaustão que se verifica em grande parte dos enfermeiros.

Os enfermeiros atravessam períodos de alta pressão a nível laboral, os quais podem potenciar o absentismo e a redução do índice de qualidade do trabalho. É recomendado o desenvolvimento de competências humorísticas por parte dos enfermeiros para aliviar o estresse no trabalho (FANG et al., 2019). O humor é uma intervenção que permite capacitar também o profissional de saúde de forma a lidar com os obstáculos, constrangimentos que existem durante o processo de cuidados (NUNES et al., 2018).

A utilização do humor pelos enfermeiros, pode ajudar a superar certas dificuldades que enfrentam no ambiente de trabalho, por outro lado, como buscam cumprir alguns objetivos sociais e se socializar humor pode ajudar nessa socialização. Essas características psicossociológicas do humor destacam-se como benefícios cognitivos e sociais expressas 
por meio de emoções positivas de alegria, da melhora da comunicação, da liberação de estresse e promoção do enfrentamento, se encontram ancoradas na ergonomia do humor como interação social (GORIUP; STRIČEVIĆ; SRUK, 2017).

\section{Fatores que influenciam o humor}

Existem diferenças de gênero no humor. Num dos estudos incluídos nesta revisão verificou-se que existem diferenças entre enfermeiros e enfermeiras quanto à apreciação, utilização do humor e humor de enfrentamento, isto é, demonstrou-se que os enfermeiros obtiveram uma pontuação mais alta do que as enfermeiras, nestas duas variáveis (NUNES et al, 2018). Estes dados parecem sugerir que o género influencia a produção e apreciação do humor.

Em estudos anteriores foram identificados alguns fatores que estão associados ou podem influenciar o humor, nomeadamente, fatores pessoais (personalidade, idade, gênero, entre outros) e os fatores contextuais/situacionais (ex.: situação clínica, gravidade da doença, experiências prévias, cultura) (SOUSA; JOSÉ, 2016; SOUSA et al., 2019a). Tendo em consideração estes fatores, o enfermeiro quando avalia, planeia e implementa a intervenção humor, depara-se com o carácter individual, pessoal e paradoxal do humor, por isso, o que é motivo de humor para uma pessoa pode não ser para outra, o momento ou situação pode não ser o mais adequado. neste sentido, tem de haver sensibilidade e bom senso na utilização do humor, pois existem relatos em que os pacientes se sentiram desconfortáveis com a situação (SOUSA; JOSÉ, 2016).

\section{Precauções na utilização do humor}

A intervenção humor em enfermagem (NIC) refere como principais restrições ao uso de humor a doença psiquiátrica agudizada (BUTCHER et al., 2018). Em estudos anteriores foi referido ainda que se deve ter em consideração que a apreciação do humor está alterada nas pessoas com Parkinson e a interpretação do humor em pessoas com alcoolismo também está afetada. Neste sentido o seu uso deve ser ponderado, pois poderá ter um efeito negativo (SOUSA; JOSÉ, 2016). O enfermeiro deve utilizar o humor com cautela pois implica treino e a adoção de estratégias individualizadas (SOUSA et al., 2019a).

\section{Implicações para a prática e educação em enfermagem}

Dado o benefício que o humor tem, para paciente e enfermeiro, as temáticas de humor na educação de enfermagem devem ser obrigatórias (GORIUP; STRIČEVIĆ; SRUK, 2017). Estes dados são suportados pelas conclusões apontadas por VAN DER KROGT et al. (2020) 
ao indicarem que o humor se apresenta como uma competência clínica e sugerem a sua incorporação em guidelines e nos currículos de ensino de enfermagem.

\section{- CONCLUSÕES}

O uso humor em enfermagem tem sido alvo da atenção de pesquisadores e enfermeiros não só pelos seus benefícios na melhoria da comunicação e relação paciente-enfermeiro como também nos efeitos positivos que este tem na saúde e bem-estar tanto nos pacientes como nos enfermeiros. É uma intervenção de baixa intensidade que faz parte das linguagens classificadas em enfermagem (NIC e CIPE), e que é sugerida em alguns diagnósticos de enfermagem (NANDA-Internacional).

Mas dado o caráter individual, pessoal e paradoxal do humor, o enfermeiro deve ter em consideração os fatores associados e os que o influenciam, nas várias etapas do processo de enfermagem. Deve-se ter em consideração ainda a situação do paciente, a gravidade da doença e a forma como este a vivencia.

Recentes estudos retratam novas intervenções baseadas no humor que visam incrementar a QV da pessoa cuidada, nomeadamente no âmbito dos cuidados paliativos e na atenção à saúde da pessoa com doença crónica.

As principais limitações desta revisão escopo estão relacionadas com a língua e com o número de base de dados incluídas. Pretendeu-se dar continuidade à revisão anterior de modo a fazer a sua atualização, por isso foram incluídos apenas artigos publicados entre 2018 e 2021.

\section{- REFERÊNCIAS}

1. ARKSEY, H.; O'MALLEY, L. Scoping studies: towards a methodological framework. International journal of social research methodology. v.8., n.1, p.19-32, 2005. Disponivel em: https://doi. org/10.1080/1364557032000119616. Acesso em: 20 mar. 2021

2. ASSOCIATION FOR APPLIED AND THERAPEUTIC HUMOR. HOME: What is therapeutic humor? Disponível em: http://www.aath.org/ (2018). Acesso em: 20 mar. 2021

3. BEHROUZ, S. et al. The Effect of Humor Therapy on Relieving Quality and Fear of Pain in Elderly Residing Nursing Homes: A Randomized Clinical Trial. Advances in Nursing \& Midwifery, [s. I.], v. 28, n. 3, p. 53-61, 2019. DOI 10.29252/anm-280309. Acesso em: 20 mar. 2021

4. BUTCHER, H. K., et al. Nursing interventions classification (NIC). St Louis: Elsevier Health Sciences, 2018.

5. Conselho Internacional de Enfermeiras. CIPE - VERSÃO 2015-CLASSIFICAÇÃO INTERNACIONAL PARA A PRÁTICA DE ENFERMAGEM. Lisboa: Ordem dos Enfermeiros, 2016. 
6. FANG, L. et al. Associations of work stress and humor with health status in hospital nurses-A cross-sectional study. Journal of clinical nursing. v. 28, n. 19-20, p. 3691-3699, 2019. DOI 10.1111/jocn.14970. Acesso em: 20 mar. 2021.

7. FIGUEIREDO, R. C., PINHEIRO, T. R. F., \& DA SILVA, M. Z. P. . O reflexo do bom humor na prática do profissional de enfermagem. Multidebates. v. 4, n.2, p.157-167, 2020.

8. GORIUP, J.; STRIČEVIĆ, J.; SRUK, V.. Is Education for Using Humour in Nursing Needed? (Slovenian Case Study on Sociological and Ergonomic Aspects of the Impact of Humour on Nursing Professionals). Acta Educationis Generalis. v. 7, n.3, p. 45-62, 2017.

9. HAWKING, S. Active Learning Introducing Therapeutic Humor. in Riley J.B. (Eds). Communication in Nursing. St Louis: Elsevier, 2019, p.137.

10. JOHNSON, M., et al. NANDA, NOC, and NIC linkages: Nursing diagnoses, outcomes, \& interventions. St. Louis: Mosby Elsevier, 2013.

11. KRISTENSEN, D. V., et al. Characteristics of Communication With Older People in Home Care: A Qualitative Analysis of Audio Recordings of Home Care Visits. Journal of clinical nursing. v.26, n.23-24, p. 4613-4621, 2017.

12. LINGE-DAHL, L. M., et al. Humor assessment and interventions in palliative care: A systematic review. Frontiers in psychology. v.9, p. 890, 2018. Disponível em: https://doi.org/10.3389/ fpsyg.2018.00890. Acesso em: 20 mar. 2021

13. MACEDO, M. A., et al.. Benefits of laughter-inducing and humor nursing interventions in people undergoing haemodialysis. Journal Aging and Innovation. v.8, n.3, p.4-20, 2019.

14. NUNES, I. R.; JOSÉ, H.; CAPELAS, M. L. Grieving with humor: a correlational study on sense of humor and professional grief in palliative care nurses. Holistic nursing practice. v.32, n.2, p. 98-106, 2018.

15. SOUSA, L. M.M. et al. Effect of humor intervention on well-being, depression, and sense of humor in hemodialysis patients. Enfermería Nefrológica, [s. I.], v. 22, n. 3, p. 256-265, 2019b. DOI 10.4321/S2254-28842019000300004. Acesso em: 20 mar. 2021

16. SOUSA, L.M.M. et al . Humor intervention in the nurse-patient interaction. Revista Brasileira de Enfermagem. Brasília, v. 72, n. 4, p. 1078-1085, 2019a. DisponivelDisponível em. http:// dx.doi.org/10.1590/0034-7167-2018-0609. Acesso em: 20 mar. 2021

17. SOUSA, L.M.M., et al. A metodologia de revisão integrativa da literatura em enfermagem. Revista Investigação em Enfermagem. S.2, n. 21, p.17-26, 2017.

18. SOUSA, L.M.M., et al. Emploi de l'humour dans la relation infirmier/personne malade: une revue de la littérature et synthèse. Revue Francophone Internationale de Recherche Infirmière. v. 4, n.1, p. 30-38, 2018a.

19. SOUSA, L.M.M., et al. Revisões da literatura científica: tipos, métodos e aplicações em enfermagem. Revista Portuguesa de Enfermagem de Reabilitação. v.1, n.1, p. 45-54, 2018 b. Disponível em: http://dx.doi.org/10.33194/rper.2018.v1.n1.07.4391. Acesso em: 20 mar. 2021

20. SOUSA, L.M.M.; JOSÉ, H.M.G. Benefícios do humor na saúde Revisão Sistemática da Literatura. Enformação. n.7, p.22-32, 2016. 
21. SOUSA, L.M.M; et al. Visualização de filmes humorísticos: repercussão na qualidade de vida na pessoa com doença renal crônica. In MISSIAS-MOREIRA,RAMON; SOUSA, LUÍS MANUEL MOTA; VALENTIM, OLGA SOUSA; JOSÉ, HELENA MARIA GUERREIRO. (Orgs). Qualidade de Vida em uma perspectiva interdisciplinar -Volume 6. (pp.31-48). Curitiba: Editora CRV, 2019c.

22. TAHERI GHARAGZLU, T.; SAFAVI, M.; FESHARAKI, M.. The effect of employee's humor training on depression, anxiety and stress of the elderly residents in Tehran's nursing homes: a randomized clinical trial. Medical Science Journal of Islamic Azad Univesity-Tehran Medical Branch, v30, n.3, p. 287-298, 2020.

23. TORRES-VIGIL, I. et al. The role of empathic nursing telephone interventions with advanced cancer patients: A qualitative study. European journal of oncology nursing: the official journal of European Oncology Nursing Society, [s. I.], v. 50, p. 101863, 2021. DOI 10.1016/j. ejon.2020.101863. Acesso em: 20 mar. 2021

24. TRICCO, A. C., et al. PRISMA extension for scoping reviews (PRISMA-ScR): checklist and explanation. Annals of internal medicine. V. 169, n.7, p. 467-473, 2018.

25. VAN DER KROGT, S. R.; COOMBS, M.; ROOK, H. Humour: A purposeful and therapeutic tool in surgical nursing practice. Nursing Praxis in Aotearoa New Zealand, [s. I.], v. 36, n. 2, p. 20-30, 2020. DOI 10.36951/27034542.2020.008. Acesso em: 20 mar. 2021.

26. ZHAO, J. et al. A meta-analysis of randomized controlled trials of laughter and humour interventions on depression, anxiety and sleep quality in adults. Journal of Advanced Nursing (John Wiley \& Sons, Inc.), [s. I.], v. 75, n. 11, p. 2435-2448, 2019. DOI 10.1111/jan.14000. Acesso em: 20 mar. 2021

27. ZHAO, J. et al. Effect of humour intervention programme on depression, anxiety, subjective well-being, cognitive function and sleep quality in Chinese nursing home residents. Journal of Advanced Nursing (John Wiley \& Sons, Inc.), [s. I.], v. 76, n. 10, p. 2709-2718, 2020. DOI 10.1111/jan.14472. Acesso em: 20 mar. 2021. 